\title{
THE QUASI-STREAMFUNCTION FORMALISM
}

\author{
Miao Tian ${ }^{1}$, William Cottrell ${ }^{2}$, Alex Sheremet ${ }^{3}$, Jane Smith $^{4}$
}

\begin{abstract}
This paper provides a review of our recent developments in reformulating the quasi-streamfunction $(\boldsymbol{\Psi})$ formalism proposed by Kim et al. (2001) to relax the common constraint of kinematic bottom boundary condition. A restricted form of the Hamilton's principle for irrotational flows is formulated only on surface variables. This transforms the problem to dynamical equations on the surface and a constraint equation related to the interior water column. The interior solution can be applied to express $\boldsymbol{\Psi}$ in terms of the natural canonically conjugate variable. The modified $\boldsymbol{\Psi}$ formalism promises to provide a natural framework for the study of wave over arbitrary bathymetry and in the presence of strong shear flow if Clebsch variables are included. We demonstrate the formalism for horizontally homogeneous flows over mild topography, where asymptotic formulations for the Hamiltonian and Lagrangian are derived. The Hamiltonian shows consistency with Zakharov's results up to the cubic order and the Lagrangian is written in terms of measurable variables.
\end{abstract}

Keywords: stream function; variational principle; hamiltonian; lagrangian

\section{INTRODUCTION}

Nonlinear wave-wave interaction in the ocean has drawn an increasing number of attention that spans a wide range of topics from the effects of wave refraction-diffraction to rogue-wave generation, wave scattering, steepening and breaking. In these topics, nonlinear coupling during wave shoaling over uneven seabed is a subject of high theoretical and practical interest because it undergoes substantial effects through this process. This yields many formalism to derive the nonlinear evolution equation of waves over a varying bottom.

In the classical approach, conservation of mass and suitable boundary conditions form the equations of wave motion within the water column. Perturbation parameters corresponding to several basic assumptions such as the locally-flat bottom or the relatively-shallow water were introduced to expand the governing equations into a perturbation series. One obtains the desired equations by keeping or discarding terms in this expansion, depending on various factors such as the wave steepness $k a$, bottom slope $\nabla h$ and the relative depth $k h$, etc. Several nonlinear wave equations, as the Hasselmann (1961; 1963a; 1963b), Agnon et. al. $(1993 ;$ 1997), the Boussinesq (1871) and some of their variations follow this classical approach.

Another approach to deriving the nonlinear wave-wave interaction equations is by use of a variational principle, especially Seliger and Whitham's (1968) variational principles in continuum mechanics. They proposed the Lagrangian formulation for irrotational flows, and also extended this principle to rotational flows by including the Lin constraint (Bretherton, 1970), which coincides with Clebsch's representation of the velocity field Clebsch (1857). Proper variational formulation for the equations of water waves were introduced by Luke (1967), Zakharov (1968; 1999), Miles (1977) and many others. All these variational principles are developed in term of the velocity potential $\Phi$, therefore they satisfy the kinematic bottom boundary condition approximately.

More recently, the quasi-streamfunction approach (hereafter $\boldsymbol{\Psi}$-formalism), first derived by Kim et al. (2001; 2003), and applied to water surface waves by Kim and Bai (2004) and Toledo and Agnon (2009), appears to hold the promise of providing a simple way to describe wave propagating over uneven bottoms with minimal restrictions. The $\boldsymbol{\Psi}$-formalism naturally defines the flow field via conservation of mass and fulfills exactly the bottom boundary condition (Kim and Bai, 2004). However, in these previous work (Kim and Bai, 2004; Toledo and Agnon, 2009), the ability to represent arbitrary slopes is eliminated by imposing the flat-bottom vertical structure to achieve mild-slope type equations. Furthermore, the variational principle is then applied to the Fourier components of the remaining horizontal variations of $\boldsymbol{\Psi}$, thus discarding the constraints arising from variations in the vertical structure.

Reformulation of this theory could transform the $\boldsymbol{\Psi}$-formalism into a useful tool for the study of wave over varying topography. We correct the slight abuse of the variational principle incurred upon ignoring

\footnotetext{
${ }^{1}$ Department of Civil and Coastal Engineering, University of Florida, 365 Weil Hall, Gainesville, FL 32611, USA. Email: mtian04.18@ufl.edu.

${ }^{2}$ Department of Physics, University of Wisconsin, Madison, 1150 University Avenue, Madison, WI 53706.

${ }^{3}$ Department of Civil and Coastal Engineering, University of Florida, 365 Weil Hall, Gainesville, FL 32611 , USA. Email: alex@coastal.ufl.edu.

${ }^{4}$ US Army Engineering Research and Development Center, Coastal and Hydraulics Laboratory, Vicksburg, MS. Email:Jane.M.Smith@usace.army.mil.
} 
vertical variations, investigate the consequences of the less constrained theory for simple horizontally homogeneous flows, and finally, understand the meaning of the function $\boldsymbol{\Psi}$. The hope is that the $\boldsymbol{\Psi}$-formalism will become a useful tool in the description of nonlinear wave-wave interaction processes in the ocean.

The governing equations are re-derived in their original lagrangian formulation without any approximations in the next section, and then solved using an expansion in powers of the free surface displacement $\eta$ in Section Homogeneous flows solutions. Alternative variational formalisms are derived in Section Hamiltonian and Lagrangian formalisms. The $\boldsymbol{\Psi}$ Hamiltonian with Zakharov's results in terms of $\Phi$ were compared and discussed. One also proposes a Lagrangian only containing measurable quantities. This work is discussed and summarized in the last section.

\section{GOVERNING EQUATIONS}

In this paper, $t$ denotes the time and the horizontal vectors are boldface, e.g., $\mathbf{x}=\left(x^{1}, x^{2}\right)=x^{1} \hat{\mathbf{x}}_{1}+$ $x^{2} \hat{\mathbf{x}}_{2}=x^{j} \hat{\mathbf{x}}_{j}$, with $j=1,2$. We will prefer Einstein's repeated-indices summation convention (last equality). The origin of the coordinate system is set on the undisturbed free surface with the vertical axis $(\hat{\mathbf{z}})$ pointing upward. The hat denotes unit vector in the direction of the axis. The free surface is defined by $z=\eta(\mathbf{x}, t)$ and the bottom by $z=-h(\mathbf{x})$. The symbol $\nabla$ denotes the horizontal gradient.

Define the quasi-streamfunction as a vector:

$$
\Psi(\mathbf{x}, z, t)=\int_{-h}^{z} \mathbf{u}(\mathbf{x}, s, t) d s
$$

The velocity field is defined as,

$$
\mathbf{u}=\Psi_{z}
$$

where $\mathbf{u}(\mathbf{x}, z, t)=u^{j}(\mathbf{x}, z, t) \hat{\mathbf{x}}_{j}$ is the horizontal velocity vector of components $u^{j}$, with $j=1,2$. From the continuity of the incompressible fluid, we obtain the vertical velocity $w$ as Kim and Bai (2004):

$$
w=-\nabla \cdot \Psi .
$$

Defining the total spatial gradient along a given surface $z=\zeta(\mathbf{x}, t)$ as

$$
\mathrm{D}_{\zeta}=\nabla \cdot+(\nabla \zeta) \cdot \partial_{z}
$$

the total divergence of $\boldsymbol{\Psi}$ on the bottom $z=-h$ is

$$
\mathrm{D}_{-h} \cdot \boldsymbol{\Psi}=-w-(\nabla h) \cdot \mathbf{u}=0,
$$

which is the standard kinematic bottom boundary condition. This equality always holds because $\left.\boldsymbol{\Psi}\right|_{z=-h}=$ 0 by (1). Therefore the quasi-streamfuntion $\boldsymbol{\Psi}$ unconditionally satisfies the kinematic bottom boundary condition (Kim and Bai, 2004).

The dynamics of the fluid system can be discussed using a variational principle. In the Eulerian description, Seliger and Whitham (Seliger and Whitham, 1968) showed that the Lagrangian density for irrotational flow is defined as the difference of the kinetic energy $T$ and potential energy $V$ plus the Lagrangian multipliers accounting for the continuity equation and kinematic constraints. The advantage of writing the Lagrangian density in terms of quasi-streamfunction is that, it is the solution of the continuity equation and kinematic bottom boundary condition. Therefore the Lagrangian $L$ and the Lagrangian density $\mathcal{L}$ are given as:

$$
L=\int \mathcal{L} d^{2} x ; \quad \mathcal{L}=\phi\left[\eta_{t}+\nabla \cdot \Psi+\boldsymbol{\Psi}_{z} \cdot \nabla \eta\right]_{\eta}+\frac{1}{2} \int_{-h}^{\eta}\left[\left|\boldsymbol{\Psi}_{z}\right|^{2}+(\nabla \cdot \boldsymbol{\Psi})^{2}\right] d z-\frac{g}{2} \eta^{2},
$$

where $\phi(\mathbf{x}, t)$ is a Lagrange multiplier for the free-surface kinematic condition (e.g., Kim et al., 2001, 2003; Kim and Bai, 2004).

One can simplify (6) significantly. Using the total derivative operator $\mathrm{D}_{\eta}$ equation (4) at the free surface $z=\eta$, the last two terms in the surface constraint becomes $\phi \mathrm{D}_{\eta} \cdot \boldsymbol{\Psi}=-\left(\mathrm{D}_{\eta} \phi\right) \cdot \boldsymbol{\Psi}=-(\nabla \phi) \cdot \boldsymbol{\Psi}, \mathcal{L}$ in (6) can be written as

$$
\mathcal{L}=\left[\phi \eta_{t}-\nabla \phi \cdot \Psi\right]_{\eta}+\frac{1}{2} \int_{-h}^{\eta}\left[\left|\Psi_{z}\right|^{2}+(\nabla \cdot \Psi)^{2}\right] d z-\frac{g}{2} \eta^{2}
$$


Moreover, the terms in the first bracket are evaluated on the free surface $z=\eta$, while the other terms are using variables within the whole water column. The term of vertical integral in (7) may be rewritten as:

$$
L_{\text {vert }}=\frac{1}{2} \iint d^{2} x \int_{-h}^{\eta} d z\left[\partial_{i} \Theta^{i}-\boldsymbol{\Psi} \cdot\left(\partial_{z}^{2} \boldsymbol{\Psi}+\nabla \nabla \cdot \boldsymbol{\Psi}\right)\right]
$$

where

$$
\Theta=\Psi \nabla \cdot \Psi+\left(\boldsymbol{\Psi} \cdot \boldsymbol{\Psi}_{z}\right) \hat{\mathbf{z}}
$$

is a 3 -dimensional vector with divergence $\partial_{i} \Theta^{i}=\nabla \cdot(\boldsymbol{\Psi} \nabla \cdot \boldsymbol{\Psi})+\left(\boldsymbol{\Psi} \cdot \boldsymbol{\Psi}_{z}\right)_{z}$. Because $\Theta=0$ on $z=-h$, applying Gauss theorem yields

$$
L_{v e r t}=\frac{1}{2} \iint_{\eta} \Theta d \mathbf{S}-\frac{1}{2} \iint d^{2} x \int_{-h}^{\eta} \boldsymbol{\Psi} \cdot\left(\partial_{z}^{2} \boldsymbol{\Psi}+\nabla(\nabla \cdot \boldsymbol{\Psi})\right) \boldsymbol{\Psi} d z,
$$

where $d \mathbf{S}=\mathbf{n} d A . d \mathbf{S}$ is the free surface element, $\mathbf{n}$ is the normal vector to the free surface, which can be written as

$$
\mathbf{n}=\frac{(-\nabla \eta, 1)}{\sqrt{1+(\nabla \eta)^{2}}} ; d A=\sqrt{1+(\nabla \eta)^{2}} d^{2} x .
$$

The first and second integrals in (10) represent an integral over the the free surface and the interior of the fluid respectively. The Hamilton's principle requires the stationary condition of the Lagrangian $L$ :

$$
\delta \int L d t=0
$$

In particular, the surface terms change independently from the interior terms, which implies that their variations mush also vanish independently. The variation of the interior term of $\mathcal{L}$ in equation (7) leads to the "Laplace"-like equation

$$
\partial_{z}^{2} \boldsymbol{\Psi}+\nabla(\nabla \cdot \boldsymbol{\Psi})=0
$$

Therefore the second integral in (10) zeros.

One finds the following simplified expression for (10) written entirely in terms of surface variables:

$$
L_{v e r t}=\frac{1}{2} \iint_{\eta} d^{2} x \boldsymbol{\Psi} \cdot\left(\boldsymbol{\Psi}_{z}-\nabla \eta(\nabla \cdot \boldsymbol{\Psi})\right),
$$

This yields a very simple expression for (7):

$$
\mathcal{L}=\frac{1}{2} \Psi^{j} \mathbf{K}_{j l} \Psi^{l}-\nabla \phi \cdot \Psi+\phi \eta_{t}-\frac{g}{2} \eta^{2} ; \text { with } \mathbf{K}_{j l}=\left(\delta_{j l} \partial_{z}-\partial_{j} \eta \partial_{l}\right)
$$

where $\delta_{j l}$ is the Kronecker symbol.

Taking variation with respect to $\eta, \phi$, and $\boldsymbol{\Psi}$, respectively:

$$
\begin{aligned}
& \eta_{t}+\mathrm{D} \cdot \boldsymbol{\Psi}=0 \text { on } \quad z=\eta \\
& \phi_{t}+(\nabla \cdot \phi) \boldsymbol{\Psi}_{z}- \frac{1}{2}\left[\left|\boldsymbol{\Psi}_{z}\right|^{2}+(\nabla \cdot \boldsymbol{\Psi})^{2}\right]+g \eta=0 \quad \text { on } \quad z=\eta \\
& {\left[\boldsymbol{\Psi}_{z}-(\nabla \cdot \boldsymbol{\Psi}) \nabla \eta+\nabla(\boldsymbol{\Psi} \cdot \nabla \eta)-\right.}\left.2 \nabla \phi+\left(\nabla \eta \cdot \boldsymbol{\Psi}_{z}\right) \nabla \eta\right] \cdot \delta \boldsymbol{\Psi} \\
&+[\boldsymbol{\Psi}+(\nabla \eta \cdot \boldsymbol{\Psi}) \nabla \eta] \cdot \delta \boldsymbol{\Psi}_{z}=0 \quad \text { on } \quad z=\eta
\end{aligned}
$$

Note that the variation $\delta \boldsymbol{\Psi}_{z}$ is treated as independent of $\delta \boldsymbol{\Psi}$ since we have a surface boundary and total derivatives can not be discarded arbitrarily.

Equations (15)-(17) together with the interior equation (12) form the governing equations of the $\Psi$ formalism in terms of $\boldsymbol{\Psi}, \eta$ and $\phi$. Equations (15) and (16) are the kinematic and dynamic surface boundary conditions respectively, and equation (17) is a constraint which relates $\boldsymbol{\Psi}$ with $\eta$ and $\phi$. No approximations have been made to equations (15)-(17) such that they are evaluated exactly at the surface.

We can retrieve the linear relationship between $\boldsymbol{\Psi}$ and $\phi$ (Kim and Bai, 2004; Toledo and Agnon, 2009) by keeping the leading order terms in the constraint equation (17). In this formalism, $\boldsymbol{\Psi}$ is corrected by higher order terms containing only one power of $\phi$ and an arbitrary number of $\eta$ 's. We will show later that, $\eta$ and $\phi$ remain canonical variables, which is useful for developing a Hamiltonian description similar to Zakharov equation (Zakharov, 1999), except that $\phi$ is not only the velocity potential at the surface. Equation (15) and (16) appear to be the dynamical equations of the system. 


\section{HOMOGENEOUS FLOWS SOLUTIONS}

Interior solutions

We solve the interior solution of $\boldsymbol{\Psi}$ using a wave-number Fourier representation. Assuming that the problem is horizontally homogeneous, the unknown functions admit wave number Fourier representation

$$
\left(\begin{array}{c}
\eta \\
\phi \\
\boldsymbol{\Psi}
\end{array}\right)=\int \frac{d^{2} k}{2 \pi}\left(\begin{array}{c}
\eta_{\mathbf{k}} \\
\phi_{\mathbf{k}} \\
\boldsymbol{\Psi}_{\mathbf{k}}
\end{array}\right) e^{i \mathbf{k} \cdot \mathbf{x}} ; \quad\left(\begin{array}{c}
\eta_{\mathbf{k}} \\
\phi_{\mathbf{k}} \\
\boldsymbol{\Psi}_{\mathbf{k}}
\end{array}\right)=\left[\begin{array}{c}
\eta \\
\phi \\
\boldsymbol{\Psi}
\end{array}\right]_{\mathbf{k}}=\int d^{2} x\left(\begin{array}{c}
\eta \\
\phi \\
\boldsymbol{\Psi}
\end{array}\right) e^{-i \mathbf{k} \cdot \mathbf{x}}
$$

where $\mathbf{k}=k^{j} \hat{\mathbf{x}}_{j}$ is the wave number vector, and the $g_{\mathbf{k}}=[g]_{\mathbf{k}}$ is the Fourier transform of $g$. The short-hand notation $[\cdots]_{\mathbf{k}}$ will later simplify the handling of convolution products resulting from the Fourier transform of nonlinear terms. Because the functions $\eta$ and $\phi$ are real, their transforms satisfy the regular symmetry conditions, e.g., $\eta_{\mathbf{k}}=\eta_{-\mathbf{k}}^{*}$ with the asterisk denoting the complex conjugate.

Substituting the Fourier representation (18) into the "Laplace-like" equation (12) for the interior flow yields

$$
\partial_{z}^{2} \Psi_{\mathbf{k}}-k^{2} \boldsymbol{\Psi}_{\mathbf{k}}=0
$$

with $k^{2}=\sum_{j} k^{j} k^{j}$ where $k$ is the absolute value of the wave number. For mildly sloping bottoms (e.g., Mei at al. (2005)), the solution of the equation for $\psi_{\mathbf{k}}$ is the usual

$$
\boldsymbol{\Psi}_{\mathbf{k}}=\frac{\sinh k(z+h)}{\sinh (k h)} \psi_{\mathbf{k}}
$$

where the wave number is assumed to be a slowly varying function of the horizontal coordinate.

\section{Perturbative surface solution}

A common approach to seek solutions for surface-gravity wave equations is to expand them evaluated at the surface in powers of $\eta$ (e.g., Zakharov, 1999). Expanding equations (15) to (17) by use of Taylor expansion up to $\mathrm{O}\left(\epsilon^{3}\right)(\epsilon \sim k a)$ yields the following expansions:

$$
\begin{gathered}
\eta_{t}+\nabla \cdot\left(\boldsymbol{\Psi}+\eta \boldsymbol{\Psi}_{z}+\frac{1}{2} \eta^{2} \boldsymbol{\Psi}_{z z}\right)=O\left(\epsilon^{4}\right) \quad \text { on } \quad z=0, \\
\phi_{t}+\nabla \phi \cdot \boldsymbol{\Psi}_{z}+\eta \nabla \phi \cdot \boldsymbol{\Psi}_{z z}-\frac{1}{2}\left[\left|\boldsymbol{\Psi}_{z}\right|^{2}+(\nabla \cdot \boldsymbol{\Psi})^{2}\right] \\
-\frac{1}{2} \eta\left[\left(\boldsymbol{\Psi}_{z}\right)^{2}+(\nabla \cdot \boldsymbol{\Psi})^{2}\right]_{z}+g \eta=O\left(\epsilon^{4}\right) \quad \text { on } \quad z=0, \\
{\left[\boldsymbol{\Psi}_{z}-\nabla \phi-\nabla(\eta \nabla \cdot \boldsymbol{\Psi})-\frac{1}{2} \nabla\left(\eta^{2} \nabla \cdot \boldsymbol{\Psi}_{z}\right)\right] \delta \boldsymbol{\Psi}+\eta\left\{\boldsymbol{\Psi}_{z}-\nabla \phi\right.} \\
-\nabla \eta(\nabla \cdot \boldsymbol{\Psi})-\eta[\nabla(\nabla \cdot \boldsymbol{\Psi})]\} \delta \boldsymbol{\Psi}_{z}+\frac{1}{2} \eta^{2}\left(\boldsymbol{\Psi}_{z}-\nabla \phi\right) \delta \boldsymbol{\Psi}_{z z}=O\left(\epsilon^{4}\right) \quad \text { on } \quad z=0,
\end{gathered}
$$

A solution for $\boldsymbol{\Psi}$ in terms of $\eta$ and $\phi$ can be obtained by substituting equation (20) into the constraint equation (23). After some algebra, the constraint equation (23) in the wave number domain yields

$$
\begin{gathered}
\left(1+m_{\mathbf{k}, \mathbf{k}_{1}}\right) \psi_{\mathbf{k}}=F_{\mathbf{k}, \mathbf{k}_{1}}, \\
m_{\mathbf{k}, \mathbf{k}_{1}}=\left[\mathbf{k}_{1} \cdot \hat{\mathbf{k}} \operatorname{cth} k_{1} h+k_{1} \operatorname{th} k h\right] \eta_{\mathbf{k}_{1}}+\frac{1}{2}\left[k_{1} \mathbf{k}_{1} \cdot \hat{\mathbf{k}}+k k_{1}+\operatorname{th} k h \operatorname{cth} k_{1} h\left(\mathbf{k}_{1} \cdot \mathbf{k}+k_{1}^{2}\right)\right]\left[\eta^{2}\right]_{\mathbf{k}-\mathbf{k}_{1}} \\
F_{\mathbf{k}, \mathbf{k}_{1}}=i \operatorname{th} k h \phi_{\mathbf{k}}+i \int \frac{d k d k_{1}}{2 \pi} \mathbf{k}_{1} \cdot \hat{\mathbf{k}} \eta_{\mathbf{k}-\mathbf{k}_{1}} \phi_{\mathbf{k}_{1}}+\frac{i}{2} \operatorname{th} k h \int \frac{d k d k_{1}}{2 \pi} \mathbf{k}_{1} \cdot \mathbf{k}\left[\eta^{2}\right]_{\mathbf{k}-\mathbf{k}_{1}} \phi_{\mathbf{k}_{1}}
\end{gathered}
$$

where $\mathbf{k}, \mathbf{k}_{1}$ are wave numbers, and using the following short-hand conventions: $[\ldots]_{\mathbf{k}}$ for the Fourier transform (18); thkh for tanh $k h$; and cth $k h$ for coth $k h$.

Equation (24) may be inverted directly to the order of accuracy required as:

$$
\psi_{\mathbf{k}}=\left(1+m_{\mathbf{k}, \mathbf{k}_{1}}\right)^{-1} F=\left(1-m_{\mathbf{k}, \mathbf{k}_{1}}+m_{\mathbf{k}, \mathbf{k}_{1}}^{2}+\ldots\right) F
$$


The procedure to write $\psi_{\mathbf{k}}$ in terms of $\eta_{\mathbf{k}}$ and $\phi_{\mathbf{k}}$ is now straightforward, albeit tedious. After some algebra, one finds the following solutions up to $O\left(\epsilon^{3}\right)$ for $\psi_{\mathbf{k}}$ :

$$
\psi_{\mathbf{k}}=i \operatorname{th} k h \phi_{\mathbf{k}}-i \int \frac{d k d k_{1}}{2 \pi} k_{1} \operatorname{th} k h \operatorname{th} k_{1} h \eta_{\mathbf{k}-\mathbf{k}_{1}} \phi_{\mathbf{k}_{1}}+i \int \frac{d k d k_{1} d k_{2}}{(2 \pi)^{2}} W_{\mathbf{k q p}} \eta_{\mathbf{k}-\mathbf{k}_{1}} \eta_{\mathbf{k}_{1}-\mathbf{k}_{2}} \phi_{\mathbf{k}_{2}}
$$

where

$$
W_{\mathbf{k k}_{1} \mathbf{k}_{2}}=k_{2}\left(\mathbf{k}_{1} \cdot \hat{\mathbf{k}}+k \operatorname{th} k h \text { th } k_{1} h\right) \operatorname{th} k_{2} h-\frac{1}{2}\left(k_{2} \mathbf{k}_{2} \cdot \hat{\mathbf{k}}+k k_{2}\right) \operatorname{th} k_{2} h-\frac{1}{2} k^{2} \operatorname{th} k_{2} h .
$$

It is noticeable that $\phi$ is equal to the velocity potential at the surface up to the leading order of (28). In the full formalism, this interpretation is corrected by higher order terms.

\section{HAMILTONIAN AND LAGRANGIAN FORMALISMS}

Within the assumptions made in the development of the approach presented here, it is possible to derive a Hamiltonian for direct comparison with existing Hamiltonian theories (Zakharov's) and a Lagarangian based on observable quantities for applications.

\section{Hamiltonian formulation}

The Hamiltonian can be transformed from Lagrangian via the Legendre transformation

$$
H=\int d^{2} x \mathcal{H}=\int d^{2} x\left(\phi \eta_{t}-\mathcal{L}\right)
$$

which yields

$$
\mathcal{H}=\nabla \phi \cdot \boldsymbol{\Psi}-\frac{1}{2} \boldsymbol{\Psi} \cdot\left(\boldsymbol{\Psi}_{z}-\nabla \eta(\nabla \cdot \boldsymbol{\Psi})\right)+\frac{g}{2} \eta^{2}
$$

An explicit form for the Hamiltonian is obtained by substituting equations (28) into (31). Because the Hamiltonian is at $z=\eta$ and the solution (28) is at $z=0$, we will expand the Hamiltonian around $z=0$ and write it as a summation of quadratic, cubic and quartic terms:

$$
H=H_{2}+H_{3}+H_{4}+O\left(\epsilon^{5}\right)
$$

where, using Zakharov's (1999) notation for convolution products,

$$
\begin{aligned}
& H_{2}=\frac{1}{2} \int d k\left(k \operatorname{th} k h\left|\phi_{\mathbf{k}}\right|^{2}+g\left|\eta_{\mathbf{k}}\right|^{2}\right) \\
& H_{3}=-\frac{1}{2} \int \frac{d k_{1} d k_{2} d k_{3}}{2 \pi}\left(\mathbf{k}_{2} \cdot \mathbf{k}_{3}+q_{2} k_{3} \operatorname{th} k_{2} h \operatorname{th} k_{3} h\right) \eta_{\mathbf{k}_{1}} \phi_{\mathbf{k}_{2}} \phi_{\mathbf{k}_{3}} \delta\left(\mathbf{k}_{1}+\mathbf{k}_{2}+\mathbf{k}_{3}\right) \\
& H_{4}=\frac{1}{2} \int \frac{d k_{1} d k_{2} d k_{3} d k_{4}}{4 \pi^{2}} T_{\mathbf{k}_{1} \mathbf{k}_{2} \mathbf{k}_{3} \mathbf{k}_{4}} \eta_{\mathbf{k}_{1}} \eta_{\mathbf{k}_{2}} \phi_{\mathbf{k}_{3}} \phi_{\mathbf{k}_{4}} \delta\left(\mathbf{k}_{1}+\mathbf{k}_{2}+\mathbf{k}_{3}+\mathbf{k}_{4}\right)
\end{aligned}
$$

and the interaction coefficient in $\mathrm{H}_{4}$ is

$$
\begin{gathered}
T_{\mathbf{k}_{1} \mathbf{k}_{2} \mathbf{k}_{3} \mathbf{k}_{4}}=V_{\mathbf{k}_{1} \mathbf{k}_{2} \mathbf{k}_{3} \mathbf{k}_{4}}+V_{\mathbf{k}_{1} \mathbf{k}_{2} \mathbf{k}_{4} \mathbf{k}_{3}} \\
V_{\mathbf{k}_{1} \mathbf{k}_{2} \mathbf{k}_{3} \mathbf{k}_{4}}=\frac{1}{2}\left|\mathbf{k}_{1}+\mathbf{k}_{4}\right| k_{3} k_{4} \operatorname{th}\left(\left|\mathbf{k}_{1}+\mathbf{k}_{4}\right| h\right) \operatorname{th} k_{3} h \operatorname{th} k_{4} h+\frac{1}{4} \mathbf{k}_{4} \cdot\left(2 \mathbf{k}_{2}+2 \mathbf{k}_{3}-\mathbf{k}_{4}\right) k_{3} \text { th } k_{3} h .
\end{gathered}
$$

where $\delta$ denotes the delta function. Comparing this to Zakharov (1999), we see that the quadratic and cubic terms are identical. As for the quartic piece, the term on the first line of equation (34) is identical while the

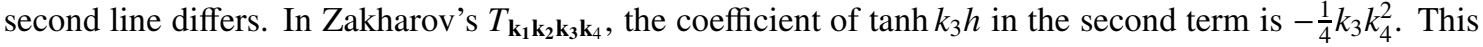
discrepancy might be due to the vector nature of $\boldsymbol{\Psi}$. At this point it is not clear if there are any measurable consequences of these differences. 


\section{Lagrangian formulation}

Based on the expansion described above, we are seeking here a Lagrangian description based on observable quantities, i.e., the generalized coordinate $\eta$ and generalized velocity $\partial_{t} \eta$. The first step is to eliminate $\phi$ by solving its equation of motion (15) for $\boldsymbol{\Psi}$ in terms of $\eta$ and $\partial_{t} \eta$. Following a similar procedure as before yields $\psi_{\mathbf{k}}$ only in terms of $\eta_{\mathbf{k}}$ and $\partial_{t} \eta_{\mathbf{k}}$

$$
\begin{gathered}
\psi_{\mathbf{k}}=\frac{i}{k}\left(\partial_{t} \eta_{\mathbf{k}}\right)-i \int \frac{d k d k_{1}}{2 \pi}\left(\hat{\mathbf{k}} \cdot \hat{\mathbf{k}}_{1} \operatorname{cth} k_{1} h\right) \eta_{\mathbf{k}-\mathbf{k}_{1}}\left(\partial_{t} \eta_{\mathbf{k}_{1}}\right) \\
+i \int \frac{d k d k_{1} d k_{2}}{(2 \pi)^{2}}\left(\hat{\mathbf{k}} \cdot \hat{\mathbf{k}}_{1}\right)\left(\mathbf{k}_{1} \cdot \hat{\mathbf{k}}_{2}\right) \operatorname{cth}\left(\mathbf{k}_{1} h\right) \operatorname{cth} k_{2} h-\frac{1}{2} \hat{\mathbf{k}} \cdot \mathbf{k}_{2} \eta_{\mathbf{k}-\mathbf{k}_{1}} \eta_{\mathbf{k}_{1}-\mathbf{k}_{2}}\left(\partial_{t} \eta_{\mathbf{k}_{2}}\right)
\end{gathered}
$$

The kinematic surface constraint becomes zero when we substitute this relationship back into equation (14) (via (18), and (20)). The lagrangian, valid up to quartic order, reads

$$
L=L_{2}+L_{3}+L_{4}+O\left(\epsilon^{5}\right)
$$

where the terms are

$$
\begin{aligned}
L_{2} & =\frac{1}{2} \int d^{2} k\left(\frac{\operatorname{cth} k h}{k}\left|\partial_{t} \eta_{\mathbf{k}}\right|^{2}-\frac{g}{2}\left|\eta_{\mathbf{k}}\right|^{2}\right) \\
L_{3} & =\frac{1}{2} \int \frac{d k_{1} d k_{2} d k_{3}}{2 \pi}\left(1-3 \hat{\mathbf{k}}_{2} \cdot \hat{\mathbf{k}}_{3} \operatorname{cth} k_{2} h \operatorname{cth} k_{3} h\right) \eta_{\mathbf{k}_{1}}\left(\partial_{t} \eta_{\mathbf{k}_{2}}\right)\left(\partial_{t} \eta_{\mathbf{k}_{3}}\right) \delta\left(\mathbf{k}_{1}+\mathbf{k}_{2}+\mathbf{k}_{3}\right) \\
L_{4} & =\frac{1}{2} \int \frac{d k_{1} d k_{2} d k_{3} d k_{4}}{4 \pi^{2}} G_{\mathbf{k}_{1} \mathbf{k}_{2} \mathbf{k}_{3} \mathbf{k}_{4}} \eta_{\mathbf{k}_{1}} \eta_{\mathbf{k}_{2}}\left(\partial_{t} \eta_{\mathbf{k}_{3}}\right)\left(\partial_{t} \eta_{\mathbf{k}_{4}}\right) \delta\left(\mathbf{k}_{1}+\mathbf{k}_{2}+\mathbf{k}_{3}+\mathbf{k}_{4}\right)
\end{aligned}
$$

and the interaction coefficient in $L_{4}$ is

$$
\begin{aligned}
G_{\mathbf{k}_{1} \mathbf{k}_{2} \mathbf{k}_{3} \mathbf{k}_{4}}=\left(\frac{1}{2}\left(\hat{\mathbf{k}}_{2} \cdot \hat{\mathbf{k}}_{3}+1\right)\left|\mathbf{k}_{2}+\mathbf{k}_{3}\right|+\hat{\mathbf{k}}_{3} \cdot \mathbf{k}_{2}+\hat{\mathbf{k}}_{3} \cdot \mathbf{k}_{3}\right)\left(1+\hat{\mathbf{k}}_{1} \cdot \hat{\mathbf{k}}_{4}\right) \operatorname{cth} k_{3} h \operatorname{cth} k_{4} h \operatorname{cth}\left(\left|\mathbf{k}_{1}+\mathbf{k}_{4}\right| h\right) \\
+\left(\frac{1}{2} k_{3}-\frac{1}{2} \hat{\mathbf{k}}_{3} \cdot \mathbf{k}_{4}+\left(\hat{\mathbf{k}}_{3} \cdot \hat{\mathbf{k}}_{3}+\hat{\mathbf{k}}_{2} \cdot \hat{\mathbf{k}}_{3}\right)\left|\mathbf{k}_{2}+\mathbf{k}_{3}\right|\right) \operatorname{cth} k_{3} h
\end{aligned}
$$

\section{DISCUSSION AND SUMMARY}

The original formulation of the $\boldsymbol{\Psi}$-formalism (Kim et al., 2001, 2003) was derived under the constraints of a flat bottom topography. An application of this work using the mild-slope approximation yields the linear CMSE (Complementary Mild-Slope Equation) (Kim and Bai, 2004) and its nonlinear extension including triad interaction (Toledo and Agnon, 2009). In these formulations, $\boldsymbol{\Psi}$ was still regarded as the linear flatbottom solution to the wave equations in term of streamfuntion, which is the product of a horizontallyvarying function $\psi(x, y)$ and a vertical structure $Z(z)$. The evolution equations were then derived by the variations of the approximated Lagrangian with respect of the horizontal component $\psi$. This study explores the possibility of reformulating the $\boldsymbol{\Psi}$-formalism to derive an exact Lagrangian without any assumptions. This Lagrangian provides a convenient framework for the study of wave over arbitrary bathymetry. In the present formalism, $\boldsymbol{\Psi}$, a $2 \mathrm{D}$-vector, allows for a one-one mapping between the surface quantities and interior solutions, at least for sufficiently small wave slopes.

Following the Lagrangian first proposed by Kim et al. (2001; 2003), a new variational $\boldsymbol{\Psi}$-formalism is derived exactly on surface. Applying variational principle to the new Lagrangian yields dynamical equations on the surface and a constraint equation related to the interior water column. The interior solution allows one to write $\boldsymbol{\Psi}$ in terms of the natural canonically conjugate variable $(\eta, \phi)$. For horizontally homogeneous flows over mild topography, we obtained an asymptotic solution for the constraint equation. A new Hamiltonian corresponding to the surface Lagrangian is derived via Legendre transformation, and expanded in terms of $\eta$. The perturbative Hamiltonian shows agreement with Zakharov's results up to the cubic order, while the different quartic Hamiltonian predicted by our approach might be attributed to the vector nature of $\boldsymbol{\Psi}$. We finally solved $\boldsymbol{\Psi}$ in terms of $\partial_{t} \eta$ and $\eta$ from the surface kinematic boundary condition and derived a Lagrangian only containing measurable quantities. 


\section{ACKNOWLEDGEMENTS}

This research was supported by the Office of Naval Research grants N00014-10-1-0805 and N0001410-1-0389.

\section{References}

Agnon, Y., A. Sheremet, J. Gonsalves, and M. Stiassnie, 1993. A unidirectional model for shoaling gravity waves, Coastal Engineering, 20, 29-58.

Agnon, Y. and A. Sheremet, 1997. Stochastic nonlinear shoaling of directional spectra, Journal of Fluid Mechanics, 345, 79-99.

Boussinesq, J., 1871. Theorie de l'Intumescence liquide appele onde solitaire ou de translation. Comptes Rendus Acad. Sci. Paris 72, 755-759.

Bretherton, F.P., 1970. A note on Hamilton's principle for perfect fluids, Journal of Fluid Mechanics, 44, 19-31.

Clebsch, A., 1857. Uber eine allgemeine Transformation der hydrodynamischen Gleichungen,J. Reine Angew. Math. 54, 293-313.

Hasselmann, K., 1961. On the non-linear energy transfer in a gravity-wave spectrum, Part I: General theory, Journal of Fluid Mechanics, 12, 481-500.

Hasselmann, K., 1963. On the non-linear energy transfer in a gravity wave spectrum, Part II: Conservation theorems - wave-particle analogy - irreversibility, Journal of Fluid Methanics, 15/2, 273-281.

Hasselmann, K., 1963. On the non-linear energy transfer in a gravity-wave spectrum, Part III: Evaluation of the energy flux and swell-sea interaction for a Neumann spectrum, Journal of Fluid Methanics, 15/3, 385-398.

Kim, J., J.W. and K.J. Bai, R.C. Ertekin, W.C. Webster, 2001. A derivation of the Green-Naghdi equations for irrotational flows, Journal of Engineering Mathematics, 40, 17-42.

Kim, J., J.W. and K.J. Bai, R.C. Ertekin, W.C. Webster, 2003. A Strongly-Nonlinear Model for Water Waves in Water of Variable Depth: The Irrotational Green-Naghdi Model, Journal of Offshore Mechanics Arctic Engineering, 125, 25-32.

Kim, J.W. and K.J. Bai, 2004. A new complementary mild slope equation, Journal of Fluid Methanics, 511, 25-40.

Luke, J.C., 1967. A variational principle for a fluid with a free surface, Journal of Fluid Mechanics, 27, 395.

Mei C.C., Mei M. Stiassnie, and D.K.-P. Yue, 2005. Theory and applications of ocean surface waves, $A d v$. Series on Ocean Eng. 23 (Expanded Edition), World Scientific.

Miles, J.W., 1977. On Hamilton's principle for surface waves. Journal of Fluid Mechanics, 27, 395.

Seliger R.L. and G.B. Whitham, 1968. Variational principles in continuum mechanics. Proceedings of the Royal Society, London, A 305, 1-25.

Toledo, Y. and Y. Agnon, 2009. Nonlinear refraction-diffraction of water waves: the complementary mildslope equations,Journal of Fluid Methanics, 641, 509-52.

Zakharov, V., 1968. Stability of periodic waves of finite amplitude on a surface of deep fluid, Journal of Applied Mechanical Technology Physics, 2, 190-198.

Zakharov, V., 1999. Statistical theory of gravity and capillary waves on the surface of a finite-depth fluid, European Journal of Mechanics, B - Fluids, 18 (3), 327-344. 